Kurt Smolak

\title{
Die Bibeldichtung Aurora des Petrus Riga (P.R.)
}

\section{Beobachtungen zu Stil und Poetik*}

Die Werkreihe der christlichen Bibelparaphrasen begann im lateinsprachigen Kulturraum des Römischen Reiches im frühen vierten Jahrhundert unter Kaiser Konstantin mit den vier Büchern Evangelia des spanischen Presbyters Iuvencus, die den vier kanonischen Evangelien entsprechen sollten, obwohl der Autor hauptsächlich das Matthäusevangelium als Referenztext verwendete, weil dieses ja am ausführlichsten ,erzählt‘. Auf Iuvencus folgten in der ersten Hälfte des fünften Jahrhunderts die fünf Bücher des Carmen Paschale des italischen (?) Presbyters Sedulius, der den narrativen vier Evangelienbüchern ein weiteres, auf das AT verweisendes gewissermaßen als Vorbereitung des soteriologischen Geschehens des NT voranstellte. Beide spätantiken Werke zählten zu den meistgelesenen poetischen Texten der folgenden Jahrhunderte. Die Werkreihe setzte sich ungebrochen bis ins Spätmittelalter fort. ${ }^{1}$ Was vornehmlich als rhetorisch beeinflusste Paraphrase begonnen hatte, sollte allmählich diverse Formen von Exegese einschließen, ${ }^{2}$ besonders allegorische Interpretationen dieser Tendenz entspricht bereits der Titel, welcher der um 500 entstandenen, ebenfalls fünf Bücher umfassenden und auf ausgewählte Episoden konzentrierten Bibeldichtung des den Arianismus bekämpfenden Alcimus Avitus von Vienne traditionell gegeben wird: 'Geistliche Geschichte', De spiritalis historiae gestis. Eine noch weiter gehende Aktualisierung eines ausgewählten biblischen Narrativs liegt in der auf die historische Situation der gegen Rom vorrückenden Truppen Belisars im Gotenkrieg Justinians applizierte Versifizierung von Abschnitten der Apostelgeschichte durch den in Rom als Subdiakon wirkenden Arator vor: Die Dichtung wurde der Überlieferung

\footnotetext{
* Als Siglen der biblischen Bücher werden jene der deutschen Einheitsübersetzung verwendet: Die Bibel, Stuttgart 2016 1547-48. - Für Hilfe bei Erstellung der diesem Beitrag zu Grunde liegenden englischen Vortragsfassung danke ich Danuta Shanzer, Wien.

$1 \mathrm{Zu}$ den einzelne Dichtungen übergreifenden Standardwerken über die Gattung als solche, deren literaturwissenschaftliche Erforschung mit dem Lexikonartikel von Thraede (1962) 997-1042 (über christliche Epik) einsetzte, zählen nach wie vor die Monographien von Green (2006); Roberts (1985); Herzog (1975): Forschungsspektrum nicht abgeschlossen; Kartschoke (1975). Darüber hinaus sei im Allgemeinen hingewiesen auf Stella 2001, besonders zur Bibelepik Smolak (2001) 15-29 (mit geringfügigen Abweichungen bereits gedruckt in: Smolak [1999] 7-24); Spezialliteratur zu einzelnen Autoren beziehungsweise einzelnen Werken wird gegebenenfalls an den jeweiligen Stellen angeführt.

2 Ein auffälliges Beispiel für die massive Einwirkung der Exegese schon auf die spätantike Bibeldichtung stellt der umfangreiche litaneiartig-anaphorische Hymnus des Dracontius auf das Licht des ersten Schöpfungstages, laud. dei 1.119-128, dar, eine freie Entfaltung des Lobes des Lichts bei Ambr. hex. I.9.33.
} 
nach im Jahr 544 in der römischen Kirche S. Petri ad vincula öffentlich rezitiert. ${ }^{3}$ Das Verhältnis des Umfangs von Referat des biblischen Bezugstexts einerseits und Kommentaren beziehungsweise Interpretationen andererseits hatte im Lauf der Weiterentwicklung der Gattung ,Bibelepik‘ sosehr überhandgenommen, dass es angebracht ist, im Hochmittelalter, konkret in der zweiten Hälfte des zwölften Jahrhunderts, weniger von Bibel-Paraphrase zu sprechen als von paraphrasiertem Kommentar.

Für die eben vorgetragene Feststellung ist die gegen Ende des zwölften und zu Beginn des dreizehnten Jahrhunderts entstandene umfangreiche bibelbezogene Dichtung des Reimser Kanonikers Petrus Riga, gestorben 1209, mit dem allegorischen Titel Aurora das am besten geeignete Beispiel. ${ }^{4}$ In ihr werden folgende biblische Bücher behandelt: der Pentateuch, Josua, Richter, Ruth, Könige 1-4 (= Samuel 1-2, Könige 1-2), Tobias, Daniel, Judith, Hester, Makkabäer, die Evangelien als Diatessaron, Apostelgeschichte, Hiob, Hohelied. ${ }^{5}$ Das ergibt eine Gesamtzahl von über 15000 Versen, leoninischen Hexametern und, der epischen Gattung im Grunde fremd, hauptsächlich elegischen Distichen, wie sie etwa zeitgleich auch Matthäus von Vendôme in seinem Tobias ebenfalls im ausgehenden zwölften Jahrhundert für ein

3 Zu Avitus s. Arweiler (1998); zu Arator s. Schwind (1990).

4 Die Tendenz des Werkes zur Kommentarparaphrase betont bereits die ausgezeichnete Edition von Beichner (1965). Beichners Text liegt den folgenden Ausführungen zu Grunde. - Zur Biographie des Autors s. Beichner (1965) XII-XVI. - Über den metaphorischen Titel, der sich in eine bereits in der Antike einsetzende, im späteren Mittelalter zunehmend dichter werdende Reihe einfügt, äußert sich der Autor im Vorwort zu der ersten Teilausgabe des Werkes: Grundlage sei die - bereits biblische (Kol 2.17 Hebr 8.5; 10.1) und frühchristliche - Metapher von ,Schatten` und ,Licht‘ für das gegenseitige Verhältnis von AT und NT einerseits und das mühevolle, mit der Morgenröte beendete Ringen Jakobs mit dem Engel (Gen 32.26) als Metapher für die Anstrengung des Dichtens andererseits (Beichner [1965] 7-8). Dass durch den Begriff Aurora ein Hinweis auf stilistischen ,Glanz' unterschwellig angedeutet wird, darf vermutet werden.- Aus frühmittelalterlicher lateinischer Bibeldichtung (tituli zu einem geplanten Bilderzyklus im Dom zu Mainz) sei die exegetische Präsentation der gesamten Bibel durch Ekkehart IV., Versus ad picturas domus Domini Mogontinae 1-4;11-12;15- 24 (zu den Schöpfungstagen 1, 4, 6) genannt, dazu s. Smolak (2015) 84-89; Leithe-Jasper (2015) 373-391; zur Bibeldichtung des Spätmittelalters s. Dinkova-Bruun (2008a) 263-284 (über paraphrastische Methoden, vorgeführt am Beispiel der Behandlung der Geschichte des Ägyptischen Joseph) und die sehr knappe Übersicht von Schmidt (2001) 449-457; vgl. auch Donnini (1995) 221-270 (über Bibelparaphrasen in diversen poetischen Gattungen 222-232, unverständlicherweise wird P.R. nicht erwähnt).

5 In seinem Vorwort äußert sich P.R. - gemäß einem schon antiken Exordial- und Bescheidenheitstopos mit Bezugnahme auf eine Aufforderung durch Freunde und anfängliche Bedenken wegen mangelnder Fähigkeit - nur auf den Pentateuch und dessen Potential allegorischer Interpretation. Es ist daher auch hinsichtlich der Ausweitung des Materials anzunehmen, dass dieser Aspekt zusammen mit der jeweils zur Verfügung stehenden exegetischen Literatur ausschlaggebend war. Das in dem zur Erweiterung offenen, nicht abgeschlossenen Werk des P.R. erkennbare Bestreben, möglichst viele biblische Bücher, im Idealfall die gesamte Schrift, zu versifizieren - die Evangelienparaphrasen des Iuvencus und Sedulius zielen nicht darauf ab, sondern behandeln die vier parallelen Texte als eine Art Diatessaron -, entspricht einer Tendenz, die sich innerhalb der spätantiken Bibelepik nur in dem fragmentarisch vorliegenden Werk des so genannten Heptateuchdichters (,Cyprianus Gallus', ,Cyprianus poeta') erkennen lässt; zu ausgewählten Aspekten dieser Bibelparaphrase s. Petringa (2016); zu der komplexen Bibel als tituli Ekkeharts IV. s. Anm. 4. 
biblisches Sujet anwendete. ${ }^{6}$ Der Bruch mit dieser linearen Tradition exegetisch-didaktisch aufgeladener bibelbezogener Dichtung erfolgte erst mit den klassizistischen Bibelepen und -epyllien des Renaissance-Humanismus. ${ }^{7}$

Die Aurora des P.R. stellt nichtsdestoweniger eine Besonderheit und daher eine Herausforderung an die philologische Analyse dar: Sie liegt nämlich als ein work-inprogress vor. Denn der Verfasser hat selbst drei Versionen erarbeitet, in denen er den ursprünglichen Textbestand im Detail erweiterte und Versifizierungen ganzer biblischer Bücher hinzufügte - diesmal in Hexametern. Dazu kommen die so genannten Recapitulationes, lipogrammatische Kurzgedichte, in denen er alttestamentliche Typologien nach Isidor von Sevilla vorstellte. ${ }^{8}$ Zum originalen Text kamen schon um 1200 die umfangreichen Erweiterungen des Aegidius von Paris, der seinerseits zwei Arbeitsgänge dafür aufwendete. ${ }^{9}$ Die breite handschriftliche Überlieferung und die zahlreichen Vorworte und Begleitschreiben aus dem Mittelalter bezeugen den hohen Grad an Akzeptanz dieser komplexen Lehrdichtung. P.R. kam offenbar einem Bedürfnis seiner Zeit entgegen. ${ }^{10}$

Im Folgenden soll versucht werden, mittels close reading verschiedene Vorgehensweisen von P.R. bei der Präsentation der versifizierten Bibel mit versifiziertem Kommentar vorzustellen. Grundlage der Untersuchung ist die Ausgabe von Beichner (s. Anm. 4). ${ }^{11}$

Als Einleitung der Ausführungen im Einzelnen diene ein Beispiel für die Art, in der P.R. mit seinem biblischen Bezugstext sprachlich-stilistisch verfährt, Ev(angelium) $409-410^{12}$ :

6 Leoninische Hexameter gebrauchte bereits Ekkehart in dem in Anm. 4 erwähnten epigrammatischen Epos gemäß mittelalterlicher tituli-Tradition, und Matthaeus von Vendôme urteilt nahezu zeitgleich mit P.R. im Nachwort seines Bibelgedichtes Tobias, 2103-2132 (2105: Tobie tituli [!]), negativ über den epischen Gebrauch.

7 Als Beispiele klassizistischer Bibelepik der frühen Neuzeit seien genannt: Marcus Marulus (Marko Marulić), Davidias (abgeschlossen 1517); Marcus Hieronymus Vida, Christias (veröffentlicht 1535).

8 Die jeweiligen Bezugsstellen aus Isidor, Allegoriae quaedam Sacrae Scripturae: Ex Veteri Testamento (PL 83,99-116) sind bei Beichner (1965) [49]-[52] aufgelistet. - Die Entstehung der recapitulationes dürfte aus dem Bestreben des Dichters erwachsen sein, technisches Können durch die lipogrammatische Versifizierung eines Prosatextes mit besonders vielen biblischen Eigennamen zur Schau zu stellen.

9 Über Aegidius s. Beichner (1965) XXI-XXIV.

$10 \mathrm{Zu}$ der Überlieferung s. Beichner (1965) L-LV: Er spricht von ungefähr 250 Handschriften. Zu späteren Handschriftenfunden (UB Heidelberg, Biblioteca Apostolica Vaticana, Bibliothek des Benediktinerklosters Engelberg) s. https://de.wikipedia.org/wiki/Petrus_Riga (abgerufen 29.1.2019); zur enormen Wirkungsgeschichte s. Dinkova-Bruun (2006) 237-260.

11 Die hilfreichen Quellenangaben von Beichner (1965) [39]-[61], können und wollen nicht mehr leisten, als einige Anhaltspunkte für weitere Interpretationen zu bieten.

12 Das Beispiel wurde ausgewählt, weil Lamech neben anderen Gestalten in der ersten exemplarischen quellenkritischen Untersuchung zur Aurora erscheint, und zwar im Genesisteil: Oppermann (1926) 60 -62. Die Namensetymologie hat P.R. im Evangelienteil nachgetragen. 
In Lamech est humilis, penetrans ad Tartara Christus;

Hinc est illud: „Ero morsus, Auerne, tuus.“

„In der Person von Lamech ist er (Christus) der Niedrigkeit des Erdbodens verbunden als einer, der in den Tartarus eindringt. Darauf bezieht sich der folgende Satz: ,Ich werde dich beißen, Unterwelts-See!““

In der Versifizierung des Stammbaums Jesu im Lukasevangelium - dargeboten mit Allegoresen der Etymologien der einzelnen Namen nach Zacharias von Besançon (Zacharias Chrysopolitanus) - findet sich bei P.R. folgende Erklärung für den Lk 3.36 erwähnten hebräischen Namen Lamech: humilis, vom Dichter versehen mit dem erklärenden Zusatz penetrans ad Tartara - dazu im Folgenden mehr. An diese Ergänzung schließt P.R. eine biblische auctoritas an: Hinc est illud: Ero morsus, Averne, tuus. Ein Zusammenhang ist zunächst nicht erkennbar, trotz des ungezwungenen Umgangs mit einem Bibelzitat, Os 13.14: ero morsus tuus, inferne. P.R. brauchte bloß das biblische Wort für Unterwelt, inferne, durch den antikisierenden Eigenamen des kampanischen Avernersees, eines der Eingänge in das Reich Plutos, ${ }^{13} \mathrm{zu}$ ersetzen und zwei Wörter umzustellen. Die Änderung des Eigennamens war übrigens aus metrischen Gründen notwendig - und nicht zu vergessen: Averne und inferne sind einander im Klang ähnlich. Außerdem konnte P.R. dadurch seine Versiertheit in der lateinischen Synonymik zur Schau stellen, schon in der Antike ein literarisches Qualitätsmerkmal: So verwendet bereits Verg. Aen. X.377-378 drei Wörter für ,Meer`innerhalb zweier Verse. P.R. glänzt in diesem Sinn innerhalb von vier Versen mit drei Ausdrücken für ,Unterwelt‘: Tartara, Averne und, zwei Verse später bereits in der Erklärung des Namens Methusalem, inferno (Ev 412, hier chronologisch anschließend im Zusammenhang mit Christi Rückkehr aus der Unterwelt).

Doch zurück zur Etymologie des Namens Lamech. Der Zusammenhang zwischen der Deutung als humilis und dem Abstieg Christi in die Unterwelt kann dem Text nicht unmittelbar entnommen werden, es sei denn man reduziert das derivierte Adjektiv auf die eigentliche Bedeutung des Substantivs humus, ,Erd(boden)‘. Für das vom Autor intendierte theologische Verständnis muss man vielmehr mit der gängigen Exegese einer anderen Bibelstelle vertraut sein: Im Christushymnus von Phil 2.8 heißt es: (Christus) humiliavit semet ipsum, factus oboediens usque ad mortem, mortem autem crucis. Die Selbsterniedrigung Christi durch seinen Abstieg aus dem Jenseits und seinen Kreuzestod bis in die Unterwelt wird schon in der patristischen Exegese häufig mit dem Zitat aus Osea als der entsprechenden alttestamentlichen Prophetie verbunden, zwar unter Weglassung des Kolons factus oboediens usque ad mortem, aber unter Beibehaltung des vollständigen Satzes des Propheten, der da lautet: ero mors tua, o mors, ero morsus tuus, inferne, z. B. Ambrosius, explanatio psalmi 43.78. P.R.

13 Avernus (Averna,- orum) ist das in der lateinischen hexametrischen Dichtung der Antike und des Mittelalters bevorzugte klassizistische Wort für Unterwelt, als Vokativ in dem angeführten Bibelzitat ist es sonst nicht nachweisbar. 
zeigt also ein Streben nach brevitas, um die biblische auctoritas in einem einzigen Vers zu konzentrieren. Dieser Absicht kam die Assonanz der Anfangswörter der zwei Kola entgegen, mors bzw. morsus.

Zum Abschluss der Interpretation von Ev 409-410 noch zur sprachlichen Formulierung des erklärenden Zusatzes zur Etymologie des Namens Lamech, humilis, dem klassisch anmutenden Halbvers von 409, penetrans ad Tartara (Christus): Diese Formulierung war zur Zeit des Autors im Zusammenhang mit Christi descensus ad inferos zwar bereits formelhaft geworden, ${ }^{14}$ hinsichtlich ihres weiteren Kontexts steht sie aber ihrer ,klassischen` Ursprungsstelle auffälligerweise in einem zentralen Detail sehr nahe, und zwar in Form einer so genannten Kontrastimitation: Ovid, met. II.260 261. Die Verse berichten nämlich von der Todesfahrt Phaëthons, des Sohnes des Sonnengottes, im Wagen seines Vaters. Er ist gewissermaßen ein ,falscher Helios‘. Als er mit dem ungezügelten Gefährt der Erde zu nahe kommt, spaltet sich der Erdboden infolge der gewaltigen Hitze, durch die so entstandenen Risse dringt das Licht in die Unterwelt ein und versetzt deren König mitsamt seiner Gattin in Schrecken: Dissilit omne solum, penetratque in Tartara rimis / lumen et infernum terret cum coniuge regem. Die Schilderung der Höllenfahrt Christi in dem weit verbreiteten, ursprünglich wahrscheinlich auf Latein verfassen Zusatz zum apokryphen Nikodemusevangelium (Acta Pilati), der descensus ad inferos, ${ }^{15}$ weist deutliche Parallelen auf: Zunächst das vom Eindringen Christi, des „Königs der Herrlichkeit“ - rex gloriae, nach ps. 23.7.9 (der Vers wird in dem Apokryphon zitiert) und somit Gegenstück zu dem ovidischen „Unterweltskönig“, infernum regem - in ihren Bereich der Finsternis in Schrecken versetzte ,Herrscherpaar‘: Tod und Teufel - im Lateinischen als Mors und Diabolus tatsächlich ein ,heterosexuelle Verbindung. ${ }^{16}$ Dazu kommt die Komponente des unverhofften Sonnenlichtes. Denn Christus wurde seit patristischer Zeit auf Grundlage der Exegese von Mal 3.20 (= 4) als die ,wahre ${ }^{6}$ Sonne der Gerechtigkeit ${ }^{17}$ verstanden. Demzufolge ist mit seinem Auftreten in der Unterwelt nicht ein ,falsches ' Sonnenlicht erschienen (wie das des Phaëthon), sondern eben jenes, das zu Beginn der Liturgie der Osternacht, für gewöhnlich knapp vor Sonnenaufgang, schon damals mit dem dreimaligen Ruf lumen Christi herbeigerufen beziehungsweise begrüßt wurde. ${ }^{18}$ Vor die-

14 Aus der paganen römischen Literatur ist noch zu nennen Lucan. 693: penetratque in Tartara lingua (sc. der Hexe Erichtho), die Stelle hat außer dem Thema ,Unterwelt keinen Bezug zu dem Bibelepos; in der christlichen Dichtung erstmals bei Sedul. carm. pasch. V.428: Tartara post caelum penetrat, post Tartara caelum, von Christi Abstieg in die Unterwelt und seiner Himmelfahrt; stilistisch und kontextuell liegt kein Bezug zu der Aurora-Stelle vor, Gleiches gilt für die späteren sprachlich ähnlichen Belegstellen.

15 Dazu s. https://de.wikipedia.org/wiki/Nikodemusevangelium (abgerufen 20.2.2019) mit Literatur. 16 Evangelium Nicodemi (Descens. Christi), 20-23 (4-7).

17 Aus der Menge der Belegstellen für Christus als, wahre Sonne' seien die früheste in Prosa und die früheste in der Dichtung hervorgehoben: Cypr. domin. orat. 35: Christus sol verus est et dies verus; Ambr. hymn. 2.5: verusque sol illabere (Bitte an Christus im Morgenhymnus).

18 Zu lumen Christi s. Dölger (1936) 1-43. - In diesen Kontext gehört auch das Konzept von Christus als der ,Übersonne‘, der nach Prud. cath. 5.127-132 durch seinen Aufstieg aus der Unterwelt im Zuge der 
sem Hintergrund, der von Gebildeten jener Epoche unschwer assoziiert werden konnte, erweist sich die Anspielung auf den Ursprung der Phrase als kontrastierende Imitation nach dem Prinzip der Ecloga Theoduli: falscher (paganer) Mythos vs. (christliche) Wahrheit. ${ }^{19}$ Die zwei genannten Punkte, Furcht der Unterweltsherrscher und Eindringen des Sonnenlichtes, zählten und zählen bis heute zu den unabdingbaren Elementen der Auferstehungsbilder vom Typ der orthodoxen Anastasis-Darstellungen, in denen die gefesselten Figuren Satans und bisweilen auch des Hades furchtsam unter den Füßen Christi neben den aufgebrochenen Höllenpforten kauern. Dieser ist umgeben von einer strahlenden, mitunter mit Sternen als Kosmos gestalteten Mandorla, die ihn in ihrer Mitte als die ,wahre' Sonne erscheinen lässt. ${ }^{20}$

Nun ein zweites Beispiel für eine zwar mühelose, aber vom Bezugstext in lexikalischer Hinsicht stärker abweichende Paraphrasierung eines Bibeltextes: Es handelt sich um den universalen Missionsauftrag Jesu an die Apostel am Ende des Matthäusevangeliums, Mt 28,18-19 bei P.R. wiedergegeben in Ev 2889-2890 und 28932896:

Discipulos post alloquitur: „mihi cuncta potestas

Est data, terrigenas caelicolasque regens“

und

\footnotetext{
„Ergo precipio vobis, gentes ut in omnes

Eloquiis vestris hec mea verba pluant,

Ut baptizetur in nomine Patris et eius

Prolis et amborum Pneumatis omnis homo.“
}

Zum Vergleich der Evangelientext: data est mihi omnis potestas in caelo et in terra ... Euntes ergo docete omnes gentes baptizantes eos in nomine Patris et Filii et Spiritus Sancti. P.R. belässt auch hier Etliches vom Wortbestand des Matthäusevangeliums, z. B. data mihi, potestas, ergo, gentes, omnes, baptizetur, in nomine Patris, setzt aber häufiger Synonyma als in den zuvor analysierten Versen- cuncta für omnis und die auf konkrete Wesen bezogenen, poetischen Komposita terrigenas und caelicolas ${ }^{21}$ für die abstrakten Raumbegriffe in caelo et in terra, wobei terrigenas auf die seit Hieronymus

Osternacht den Tag zurückbringt, s. Smolak (1994) 32-33. Dass in der solaren Komponente des descensus ad inferos Einfluss von Mysterienkulten (Isis) vorliegt, ist nicht auszuschließen, vgl. Apul. met. 11.23 .

$19 \mathrm{Zu}$ Methode und Zielsetzung dieses bukolischen Gedichts s. Herren (2007) 190 - 230.

$20 \mathrm{Zu}$ den Anastasis-Darstellungen s. Loerke (2003) 26-36; ferner www.beyars.com/kunstlexikon/le xikon_4133.html (abgerufen 23.3.2019). - Besonders deutlich ist die solare Komponente Christi als Kosmokrator in der Unterwelt dargestellt in dem Anastasis-Fresko im Parekklesion der Chora-Kirche (Kariye Camii) in Konstantinopel / İstanbul aus 1320/21.

21 Ms Cambridge, Gonville and Caius College Library, 363.569 (CMA 1068) kehrt die Wortstellung entsprechend jener des Bibeltexts um: celicolas terrigenasque. 
gängige Etymologie von Adam, terrigena, verweist. ${ }^{22}$ Das über den Bezugstext überschießende, syntaktisch auffällige, da auf potestas und nicht auf den Sprecher bezogene Partizip regens in Ev 2890, in der Überlieferung denn auch durch rego ersetzt, ${ }^{23}$ soll auf das Königtum des auferstandenen und in den Himmel zurückgekehrten Christus anspielen, dem potestas „gegeben wurde“, data est. Die Verbalform des passiven Perfekts scheint der Dichter im Sinn des historischen, d. h. innerhalb der Zeit vollzogenen Aktes zu verstehen, nämlich der Inkarnation oder der Beglaubigung durch die in allen kanonischen Evangelien bezeugte Hallstimme des Vaters bei der Taufe Jesu im Jordan. Durch die Ausformulierung dieser Exegese in dem folgenden Distichon 2891-2892 verwahrt sich P.R. gegen die Annahme eines außerzeitlichen Zustandsperfekts, das eine Subordination des ,Empfängers‘, also des Sohnes, unter den ,Gebenden', also den Vater, im Sinn des Arianismus nahelegen würde. ${ }^{24}$

Die auf diese christologische Exegese folgende sprachliche Gestaltung des Missionierungsauftrags in 2893-2894 ist in die Metapher eines befruchtenden Regens gekleidet und für die Benetzung der Menschen mit den Worten Christi verwendet. Es handelt sich hier nicht um ein bloß poetisches Schmuckmittel, sondern die Metapher soll zunächst den unmittelbar folgenden Befehl Christi zur Taufe in der damals längst üblichen Form einer Infusionstaufe durch Stiftung einer Assoziation lexikalisch vorbereiten. Der ,Regen der Worte Christi‘, vermittelt durch die ,(kultivierten) Reden` der Apostel, meint also die der Taufe vorangehende Taufkatechese, die Petrus nach Apg 2.38 empfiehlt, und auf die er in dem pseudepigraphischen so genannten zweiten Petrusbrief, 2 Petr 1.5-11, Bezug zu nehmen scheint. ${ }^{25}$ Die apostolischen Katecheten werden in dieser ihrer Funktion als Weise gemäß einer Definition in Sir 39.9 (Zählung der Vulgata) und dadurch als Vollender des AT vorgeführt: Von dem auf Gotteser-

22 Tert., adv. Marc. II.12.3 als Attribut von animalia in Gegensatz zu aquigena; Hier nom. hebr. p. 73,1.23 Lagarde als alternative Übersetzung von Adam neben homo. - Der früheste Beleg für das Wort findet sich in einem unsicheren Fragment bei Lucil. 1377 Marx = D 35 Charpin (überliefert von Cic. div. 2.133) in der bekannten parodistischen Periphrase für die Schnecke; als poetisches Synonym für Menschen erstmals gebraucht von Lucr. 5.1114. Eine nur scheinbare Ausnahme stellt die Verwendung in einem nicht poetischen Text bei Flor. epit. 1.31.6, dar: Das Wort ist dort als die Übersetzung des griechischen

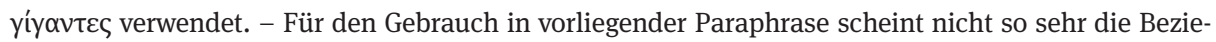
hung auf Adam als vielmehr der poetische Charakter und die Übereinstimmung mit celicolas in Umfang, Rhythmus und Vokalharmonie maßgeblich gewesen zu sein: Dem Begriff fehlt hier nämlich jegliche negative Konnotation, die er bei christlichen Autoren nicht selten hat.

23 Ms Oxford, Bodleian Library, Laud Misc. 576.

24 Hier der Text: Hoc de carne sua dicit, non de Deitate, / Qui Patri consors consimilisque manet. - Die vorgetragene Erklärung findet sich weder in der Historia Scholastica noch bei Zacharias von Besançon (Zacharias Chrysopolitanus), dessen Kommentar P.R. über weite Strecken folgt, scheint also von dem Dichter selbst entwickelt, s. Beichner (1965) II, [48], möglicherweise in Anlehnung an die Unterscheidung zwischen menschlicher und göttlicher Natur Christi im Zusammenhang mit der Aussage des Auferstehungsengels in Ev 2863-2864, dass der Auferstandene „nicht hier“ sei; die Quelle für diese auf die Zwei-Naturen-Christologie bezogene Exegese dieser Stelle findet sich bei Zacharias (PL 186,591C).

25 Vgl. Ruf (2011) 208. 
kenntnis ausgerichteten Weisen heißt es nämlich an der alttestamentlichen Stelle: tamquam imbres mittet eloquia sapientiae suae - als Signalwort fungiert eloquia, unterstützt durch das von P.R. mit dem synonymen pluat übernommene Bild des Regens. Sowohl der Weise im Sinne der Sirachstelle als auch die Apostel verkünden demnach in ihren Reden dieselbe Weisheit, nämlich die ihnen von Gott übermittelte: Die Sapientia (Dei) nimmt ja gemäß Weish 1.4 nur im Herzen des Rechtschaffenen Wohnung. Die Metapher des (befruchtenden) Regens für die Katechese leitet außerdem zur Assoziation zu einem anderen Metaphernkomplex über. Denn die Bereitschaft zur Taufe kann im Sinn des Sämannsgleichnisses von Mt 13.8 als reiche Feldfrucht der Annahme des Wortes Gottes gesehen werden. Reiche Ernte setzt aber genügend Bewässerung durch Regen voraus. Der Auftrag, diese Ernte einzubringen, ist wie an der biblischen Bezugsstelle in 2895-2896 als trinitarische Taufformel ausgesprochen: Ut baptizetur in nomine Patris et eius / Prolis et amborum Pneumatis omnis homo. Die trinitarische Taufformel bleibt durch den Ersatz von Filius durch proles auf poetischem Niveau. Dasselbe kann auch für das griechische Wort Pneumatis statt Spiritus gelten - auch an anderen Stellen der Aurora dienen Pneuma und Flatus entsprechend einer lexikalischen Tradition lateinischer christlicher Dichtung als Synonyme für den (Heiligen) Geist. ${ }^{26}$ Allerdings dürfte in dem Zusatz amborum auch eine leise polemische Anspielung auf die Ostkirche enthalten sein, die ja das Filioque des Chalzedonensischen Konzils nicht angenommen hat und daher vom westlichen Standpunkt aus nicht ,orthodox' im ursprünglichen Sinn des Wortes ist. Der Zusatz amborum geht also über die sprachliche Gestaltung des Referenztextes hinaus und enthält eine dogmatisch-theologische Dimension.

Im Folgenden sollen kurz weitere Beispiele formaler und / oder inhaltlicher Modifikationen des biblischen Berichts vorgeführt werden, die stärker als die bisher analysierten in diesen eingreifen und ihn somit verändern. Dabei ist stets zu bedenken, dass P.R. nicht eine bloße Bibelparaphrase bieten will wie die frühen patristischen Bibelepiker von sich behaupteten ${ }^{27}$ - obwohl auch sie ihre Bezugstexte ver-

26 Flatus wird in der patristischen Prosa häufig für das Wirken (,Wehen') des Heiligen Geistes gebraucht, in der Dichtung besonders von Iuvencus, bei dem der Begriff zum Synonym von spiritus erscheint: II.714; IV.97, bei P.R. vgl. Ev 17: Dona Sacri Flatus.

27 Besonders ,Cyprianus Gallus‘ (Heptateuchdichter) will nur paraphrasieren und enthält sich exegetischer Zusätze, trotzdem werden sachliche Änderungen allein durch das Metrum und die epische Sprache erzwungen, z. B. bereits im ersten Vers Gen 1-2: Principio dominus caelum terramque locavit: / namque erat informis fluctuque abscondita tellus (Gen 1.1: In principio creavit Deus caelum et terram. Terra autem erat inanis et vacua et tenebrae super faciem abyssi et spiritus dei ferebatur super aquas). Es wird nicht nur der erst im Elohistenbericht angewendete Machttitel dominus (deus) als Ersatz für Deus verwendet, sondern durch das Prädikat locavit sogar der Akt der creatio ex nihilo (unbewusst?) durch eine Trennungs- (Diakrisis-) Kosmogonie im antiken Sinn unter Weglassung der facies abyssi ersetzt, so dass die Aussage eher Ov. met. I.21-22 (hanc deus et melior litem natura diremit; / nam caelo terras et terris abscidit undas) nahesteht als jener der Bibel.- Methodisch grundlegend dazu Herzog (1975) 109123. Zum formalen Aspekt der sprachlichen Transposition bei dem Heptateuchdichter, ohne detaillierte Interpretationen s. Petringa (2016) 29-67; aber bereits Iuvencus, der anders als der Heptateuchdichter 
änderten. Er beabsichtigt vielmehr eine der aktuellen Exegese folgende Kommentierung der von ihm ausgewählten biblischen Bücher in ansprechender Form zu verfassen. Eine seiner Hauptquellen ist hierfür, wie Ende des zwölften Jahrhunderts nicht anders zu erwarten, die für den Pariser Studiengang verfasste Kirchengeschichte Historia scholastica des Petrus Comestor. ${ }^{28}$ Auf sie muss in vorliegender Studie mehrfach hingewiesen werden. Die drei folgenden Abschnitte sind alle dem ersten Schöpfungsbericht, Gen 1.1-2.3 (,Priesterbericht'), entnommen, einem der von der Patristik am ausführlichsten kommentieren biblischen Narrativen überhaupt. Trotz der durchgehenden Bezugnahme auf exegetische Referenztexte verfährt der Dichter in Gewichtung und Anordnung des Materials im Detail vergleichsweise frei. ${ }^{29}$

Erstes Beispiel: Erster Schöpfungstag (Gen 7-8 P.R. ${ }^{30}$ )

Principio celum terramque creasse refertur

Qui sine principio, qui sine fine manet.

Gleich die erste sprachliche Einheit, in principio beziehungsweise in der dichterischen, seit der Welterklärung durch Anchises bei Verg. Aen. 6.724 präpositionslosen Form principio, unterzieht P.R. einer christologischen Deutung, die freilich modernen hermeneutischen Kriterien nicht standhalten könnte. Doch vorab müssen zwei Be-

für seine Paraphrase ornamenta terrestria linguae (IV.805) zulässt, induziert raffiniert den Bibeltext überhöhende Exegese: Wenn er den Besuch der Frauen am Grab Jesu mit einem epischen Morgenanbruch einleitet, ersetzt er die biblischen Ausdrücke quae lucescit in primam sabbati (Mt 28.1), valde mane ... orto iam sole (Mk 16.2), valde diluculo (Lk 24.1), mane, cum adhuc tenebrae essent (Jh 20.1) in 4.743 durch sidera iam noctis venturo cedere soli / incipiunt, und weist unter Nutzung des exegetischen Potentials der diversen Lichtelemente aller vier Zeitangaben (von ,noch dunkel` bis ,nach Sonnenaufgang ${ }^{\star}$ ) mittels des Futurpartizips venturo, das in epischer Sprache für den Sonnenaufgang nicht üblich und etwa surgenti (Verg. Aen. XII.172: surgentem ... solem) metrisch gleichwertig ist, auf die unmittelbar bevorstehende Auferstehung Christi hin, welcher z.B. Chromat. in Matth. 54 A,1.68 und Ambr. epist. IX.68 als venturus sol iustitiae gemäß der christologischen Exegese von Mal $3.20=4.2$ : oriteur ... sol iustitiae bezeichnet wird - venturus wird ferner in Glaubenssymbolen für die zweite Parusie Christi verwendet. - Naturgemäß müssen überdies Bibelcentonen auf größtmögliche Nähe zum Referenztext verzichten: $\mathrm{Zu}$ den diesbezüglichen Problemen des Cento s. schon Herzog (1975), Smolak (2004a) 55-56.

$28 \mathrm{Zu}$ den Quellen der Aurora s. Beichner (1965) XLVII-L. Auf Petrus Comestor als wichtigste Informationsquelle hat erstmals Oppermann (1926) 55-73 hingewiesen.

29 Als Beispiel diene die insgesamt 36 Verse umfassende Präsentation des ersten Schöpfungstages: Das die Handlung des Sechstageberichts bestimmende ,Machtwort‘ Gottes, fiat, sc. lux (Gen 1.3), wird erst im Zusammenhang mit der ,Erhellung، der alttestamentlichen Schriften durch den von Jesus (29: veniente Iesu) gesandten Heiligen Geist angedeutet, der bis dahin nur „über den Wassern schwebte“ (Gen 1.2), offenbar ohne sie zu „befruchten“. Das ,Machtwort“ ist somit chronologisch von der Erstschöpfung getrennt, als Metapher syntaktisch in Form einer Partizipialkonstruktion (31: facta luce) marginalisiert und des Sprechcharakters beraubt. Die Stelle der Lichtschöpfung besetzte P.R. dagegen durch die Angabe der schon in der vorchristlichen Exegese in den Bibelbericht eingedrungenen Erschaffung der Himmelswesen (15-16).

30 Zur Vermeidung von Unklarheit bei Zitaten der Genesisparaphrase von dem paraphrasierten Bezugstext sind ersterer jeweils die Initialen P.R. beigefügt. 
merkungen über die Zusammenfassung aller geschaffenen himmlischen Wesen unter dem Begriff celum durch P.R. und deren Folgen gemacht werden: 1. Für diese Begriffserweiterung dient ein argumentum ex silentio als Beweis: Die zugleich mit dem Himmel erschaffene Erde sei am ersten Tag noch „wüst und leer“ gewesen, über den gleichzeitig geschaffenen Himmel werde das aber nicht ausgesagt. Daraus sei zu schließen, dass der Himmel bereits von den Engeln besiedelt gewesen sei (Gen 13-14 P.R.: non ita de celo scriptum legis ut sit inane, / quod replet angelicus, ditat et ornat honor). 2. P.R. interpretiert in principio nicht in temporalem, sondern in instrumentalem Sinn. ${ }^{31}$ Als instrumentale Präposition entspricht in dem weit verbreiteten instrumentalen griechischen '́v der Septuaginta. ${ }^{32}$ Somit ist (in) principio gleichbedeutend mit in Christo, „durch (den präexistenten) Christus“, das Wort Gottes, den Logos „vom Anfang“, gemäß dem Johannesprolog, Jh 1.1. Jesus bezeichnet sich übrigens Jh 8.25 selbst als principium. Diese von P.R. als bekannt vorausgesetzte Exegese führt beispielsweise Aug. gen. c. Manich. I.2.3 klar aus: Deum [in principio] fecisse caelum et terram, non in principio temporis, sed in Christo, cum verbum erat apud patrem, per quod terra et in quo facta sunt omnia. Dominus enim noster Iesus Christus, cum eum Iudaei interrogassent, quis esset, respondit: principium, quod et loquor vobis. P.R. selbst bekräftigt seine anfänglich wenig deutliche Interpretation von principium, indem er nur einige Verse später, Gen 21-22 P.R., ausdrücklich schreibt: Principium Iesus est, celum creat Auctor in isto,/ Per quem celestes efficit esse viros.

Da aber in Christus, kraft dessen der Schöpfer den Himmel hatte entstehen lassen, auch die Kirche ihren Ursprung hat, repräsentiert die noch leere Erde eben die erst zu erschaffende, aber vor der Zeit von Gott konzipierte Kirche (Gen 23 P.R.): Terra prius vacua notat ecclesiam sine fructu. Die Zweckbestimmung der Kirche, beziehungsweise die ihres Schöpfers Christus, sei es aber, „himmlische Männer“, celestes viros (Gen 22 P.R.), das bedeutet: heilige, einer himmlischen Existenz würdige Männer (!), hervorzubringen. Dieses Konzept macht den Eindruck einer Applikation der hocharchaischen Vorstellung der ,Heiligen Hochzeit‘ von Himmel und Erde als dem Ausgangspunkt allen Seins, in der europäischen Tradition durch den schon von Hesiod, theog. 45-46 bezeugten Mythos der Vereinigung von Uranos und Gaia belegt. Wohl in Hinblick auf die Vorstellung einer ,Vermählung“ verwendet der Dichter Gen 24 P.R. das Verb iungere: Donec Christus adest et sibi iungit eam (sc. ecclesiam), eben als con-iunx, seine „Gattin“, offenkundig eine Bezugnahme auf die weit verbreitete allegorische Exegese des Hoheliedes, der gemäß die Kirche die Braut Christi sei. ${ }^{33}$

31 An der zitierten Vergilstelle hat das präpostitionslose principio wohl unter dem Einfluss der didaktisch-deduzierenden Diktion des Lukrez nicht zeitliche, sondern enumerativ-argumentative Funktion, in biblisch-zeitlicher Funktion verwendet es ,Cyprianus Gallus‘ (Heptateuchdichter) Gen 1, s. Anm. 27.

$32 \mathrm{Zu}$ diesem Gebrauch von in s. Hofmann, Szantyr (1965) 126.

33 Z. B. Greg. M. in cant. 8.: dominus et ecclesia ... sponsus nominatur et sponsa, 10: sponsa ... ipsa perfecta ecclesia est; Bern. Clarav. serm. super cant. 14.7: sponsa ... ecclesia est, 25.9: sponsus ecclesiae Iesus Christus. - Die Vermählung von Christus und Ecclesia ist nach der Historia Scholastica (PL 
In Weiterführung der Allegorese der christianisierten ,Heiligen Hochzeit' handelt Gen 25 P.R. von der dieser Verbindung entstammenden Nachkommenschaft: huius (sc. Christi) in adventu datur ecclesie nova proles. Die Phrasierung des Verses lässt erkennen, dass der Dichter auf der im 12. Jahrhundert allgemein akzeptierten Deutung der vierten Ekloge Vergils auf die Ankunft Christi in seiner irdischen Geburt aufbaut, wie sie der Überlieferung nach Kaiser Konstantin vor den Konzilsvätern von Nizäa im Jahr 325 vorgetragen hat. ${ }^{34}$ In Abweichung von der Tradition der durchgehend christologischen Interpretation teilt P.R. den Vers Verg. ecl. 4,7: iam nova progenies caelo demittitur alto auf Christus als den vom Himmel kommenden Messias, huius in adventu, und das „,neue Geschlecht“ der zu errettenden Menschheit, nova proles, auf, das der bis dahin ,ertraglosen“, sine fructu, das bedeutet metaphorisch ,unfruchtbaren“ Erde entsprossen war - die allgemein geläufige Etymologie von Adam als „Erdensohn“, terrigena (s. Anm. 22), kommt dieser Metaphorik entgegen: der ,Neue (,letzte', ,endgültige') Adam‘, novissimus Adam, bei Paulus 1 Kor 15.45 Christus selbst, ist hier auf die Menschheit als ,Neue Adamsnachfahren', nova proles, übertragen.

Zweites Beispiel: Vierter Schöpfungstag (Gen 88-102 P.R.)

Sol et luna duo lumina magna micant;

Noctem sive diem distinguunt hec duo; fiunt

Nota per hec anni tempora, signa, dies.

Sol notat ecclesiam, synagogam luna figurat;

Vel sol esse potest lex nova; luna, vetus.

Nullum luna iubar sine solis lumine profert:

Nil prodesse potest lex vetus absque nova.

Vel sol doctores preclaros, luna minores

Signat, qui spargunt lumen in orbe suum.

Mox ut preficitur luci sol cereus orbis,

Et lune speculo nox radiata nitet,

Tamquam candelis non sic ardentibus, astris

Innumeris celi sphera nitere datur.

Astra notant iustos qui qualemcumque nitorem

Dant populis iuxta posse modumque suum.

In der Paraphrase des vierten Schöpfungstages fällt zunächst die allegorische Interpretation von Sonne und Mond als Kirche und Synagoge auf. Grundlage dafür ist die schon in der Antike bekannte Tatsache, dass der Mond sein Licht von der Sonne erhält. ${ }^{35}$ Diese Erkenntnis hatte bereits Ekkehart IV. in seinem epigrammatischen Bi-

198,1070B) auch in den Prophetien Adams (vgl. Anm. 44) enthalten, auf die sich P.R. in Gen 309-310 P.R. ohne konkrete Inhaltsangaben bezieht - oder sollte er mit fidem auf die eheliche Treue der Braut anspielen, die den ,rechten Glauben' bewahrt?

34 Der Abschnitt mit der Rede Konstantins Ad sanctorum coetum über die Sibyllinischen Orakel und Vergil, ecl. 4, als christliche Prophetien (Eus. HE 4.28, über Vergil XIX-XXI) gilt heute nicht mehr als authentisch und ist daher in neueren Ausgaben nicht enthalten; gesondert ediert von v. Heikel (1902).

35 Z. B. Lucr. V.705; Cic. nat. deor. II.119; rep. VI.17; Ambr. hex. IV.2.7. 
belepos verwertet, aber ohne Allegorese. ${ }^{36}$ Für P.R.s allegorische Deutung bietet die Abhängigkeit der Gültigkeit des dunklen, das heißt nur in ,Typen` das Heilsgeschehen verborgen andeutenden Alten Testaments von dem hellen, das heißt die Ereignisse in ihrer Erfüllung offenbarenden Neuen Testament, die Grundlage für die Allegorese der zwei ,großen Leuchten‘. Dieselben Qualitäten, nämlich dunkel und hell, werden übrigens in der zeitgleichen Stiftungsinschrift des so genannten Verduner Alters im Chorherrenstift Klosterneuburg bei Wien den zwei Testamenten zugewiesen, die ja de facto Synagoge und Kirche entsprechen. ${ }^{37}$ In einem weiteren Ansatz der Allegorese bezieht P.R. die unterschiedliche Helligkeit der Himmelskörper auf Personen mit unterschiedlich intensiver geistlicher und theologischer ,Strahlkraft', ,Kirchenlichter - die laut Bibelbericht zuletzt geschaffenen Sterne bezieht er auf ,Gerechte‘, das heißt heiligmäßige Personen, ungeachtet der Tatsache, dass es Mt 13.43 heißt, „die Gerechten“ - ohne Differenzierung - würden einst „im Himmelreich wie die Sonne strahlen“.

Lexikalisch bemerkenswert innerhalb der Paraphrase des vierten Schöpfungstages ist aber gerade die Metapher für die Sonne: cereus orbis (Gen 97 P.R.). Sie passt zwar in den umgebenden astralen Metaphernkomplex, ist aber dennoch ungewöhnlich und lexikalisch bemerkenswert, stiftet sie doch nahezu notwendigerweise bei den Lesern die Assoziation zur Osterkerze, für die cereus mit oder ohne das Attribut paschalis die terminologische Bezeichnung ist. ${ }^{38}$ Gemäß der Allegorese von P.R. wären demnach durch die Sonne die vier ,großen` westlichen Kirchenväter, doctores preclaros (Gen 95 P.R.), repräsentiert, durch den Mond die nachantiken, von den patres abhängigen Kirchenlehrer wie etwa Beda Venerabilis, minores (Gen 95 P.R.), zum Unterschied von den noch kleineren Leuchten, wohl Lokalheiligen mit begrenzter Strahlkraft, qualemcumque nitorem / dant populis (Gen 101-102 P.R.), welche von den „nicht so (stark) glühenden“ Sternen / Kerzen, candelis non sic ardentibus (Gen 99 P.R.), repräsentiert werden ${ }^{39}$ - candela bezeichnet, zum Unterschied von cereus, stets eine kleine, handliche Kerze. Am auffälligsten an der Behandlung des vierten Schöpfungstages ist aber, dass P.R. die naheliegende, seit frühesten Zeiten geläufige

36 Versus ad picturas 11-12: zu dem Werk s. Anm. 4 und Smolak (2015) 86-87.

37 Die Stifterinschrift von 1182 enthält folgende wertende Dunkel-Hell-Metaphern zu den zwei Testamenten (vgl. Anm. 4): umbre (3), obscura (5) über das AT, pura (6) über das NT. Der Text findet sich z.B. bei Buschhausen (1980) 13 mit (fehlerhafter) deutscher Übersetzung; Korrekturen dazu Buschhausen (1987) 265-309 (in Kooperation mit Smolak); als nova lex bezeichnet z. B. eine Inschrift auf dem so genannten Wiltener Kelch (Wien, Kunsthistorisches Museum, Inv. Nr. 8924) von 1160/70 das NT, s. Fillitz, Pippal (1987) 171-172; zum Ausdruck lex nova für die Ära des Evangeliums vgl. bereits Paul. Nol. epist. 32.5; carm. 22.155.

38 Zum Begriff cereus, wahrscheinlich für ,Osterkerze‘, vgl. Aug. civ. XV.22; vom Mittelalter an ist cereus (paschalis) jedenfalls terminus technicus für die Osterkerze.

39 Zur Metapher lumen ecclesiae, 'Kirchenlicht' für Theologen: Mar. Victorin. adv. Arrium 1 A 28 (von Konzilsvätern von Nizäa); Cassiod. in psalm. SL 97, psalm. 9.1.645 (Hilarius und Augustinus), in Mittelalter und Neuzeit häufig belegbar, im Deutschen mitunter noch in der Gegenwartssprache mit Negation versehen für intellektuell minder begabte Personen in Gebrauch. 
Interpretation der Sonne als Symbol Christi, nach der allegorischen Auslegung der Stelle Mal 3.20 (=4.3) der ,Sonne der Gerechtigkeit‘, nicht bietet. Vielmehr beschränkt er sich, wie bereits erwähnt, auf die Exegese der Beziehung zwischen AT und NT in Hinblick auf die Lehrautorität der Kirche. Der Grund dafür muss in den vorgestellten Adressaten des Werkes liegen, nämlich zölibatär lebenden Klerikern, für welche die Dichtung eine mit Genuss lesbare Belehrung sein soll. Dies geht aus den Versen Gen 163-166 P.R. hervor. ${ }^{40}$ Dort wird Gottes Befehl an die Ureltern, sich zu vermehren, von den Lesern ausdrücklich ferngehalten, indem die ,Vermehrung' in Allegorese zunächst allgemein auf menschliche Ethik (zweimal dicitur his) bezogen wird und an zweiter Stelle, wie aus dem Zusammenhang hervorgeht, im Besondern auf das Wirken einer eigens apostrophieren (tibi dictum) ausgewählten Gruppe von Lehrenden in der Kirche. Jeder Bezugnahme auf körperliche Fortpflanzung wird mit sprachlichen Mitteln unterbunden, möglicherweise auf Grund der von den Kirchenvätern, besonders von Augustinus vertretenen Auffassung einer ohne als Lust empfundenen, sondern dem Willen und dem Pflichtbewusstsein gegenüber dem Auftrag Gottes zur Fortpflanzung unterworfenen sexuellen Betätigung vor dem Sündenfall. ${ }^{41}$ Soweit die in sich geschlossene Allegorese des P.R. Das Fehlen einer In-Beziehung-Setzung von Sonne und Christus wurde aber schon auf der frühesten Rezeptionsstufe der Dichtung als Mangel empfunden: Der „letzte Korrektor“, ultimus corrector, in Wahrheit erweiternde Bearbeiter der Aurora, der eingangs erwähnte Aegidius von Paris, hat bereits in seinem ersten Revisionsdurchgang die Allegorese der Sonne auf Christus als eine weitere Erklärungsmöglichkeit nachgetragen. ${ }^{42}$

Als drittes und letztes Beispiel für die auf Exegese gegründete Behandlung des Priesterberichts des Sechstagewerkes Anmerkungen zum sechsten Tag, dem Tag der Menschenschöpfung. Die Darstellung der Erschaffung von Adam und Eva ist in der Aurora wie in der Bibel fragmentiert, und das noch deutlicher als in dem Bezugstext: Sie erscheint nämlich bei P.R. zunächst in der interpretierenden Paraphrase des Priesterberichts mit deutlicher sprachlicher Bezugnahme auf diesen (Gen 133-176 P.R.). Die narratio ist verbunden mit trinitarisch-theologschen und anthropologischen Fragen - ausgehend von dem Plural faciamus (hominem) von Gen 1.26, dem Problem des Ebenbildes Gottes im Menschen, der Herrschaft des Menschen über die Natur und endend mit der Frage nach der Abwesenheit des - im ersten Schöpfungsbericht bereits oder noch nicht - gestürzten Satan / Luzifer. Diese Frage bietet zugleich eine Überleitung zum zweiten, gemäß der biblischen Abfolge erst nach der Sabbatruhe Gottes

40 Hier die Verse Gen 163-166 P.R.: „Crescite,“ dicitur his, „et multum reddite fructum.“ / Pertinet ad mores ista loquela bonos. / Dicitur his: „Terram replete.“ Nota tibi dictum: / Plenus in ecclesia fructibus esto bonis. Die Interpunktion der Ausgabe von Beichner (1965), Semikolon nach dictum, ist irreführend. 41 Ausdrücklich auf den Zustand der Menschen im Paradies bezogen Aug. c. Iul. 4 (PL 44,757 A); vgl. Anm. 46.

42 Nach Vers 94 ergänzte Aegidius 1.1-6: Vel sol est Christus, et lux que sole relucet / Ecclesiam signat ecclesieve fidem. / Preficitur luci sol, cereus orbis: ab illo / Lux nitet, a Christo fit radiosa fides. / Vere sol Christus, sine quo nox occupat omnes, / nec recte fidei lucet in orbe dies. 
(Gen 177-188 P.R.) behandelten Schöpfungsbericht. Dieser enthält erwartungsgemäß die zweite Beschreibung der Erschaffung der protoplasti. Diese ist ihrerseits aufgeteilt in eine gesonderte narratio der Bildung von Adams Leib aus Lehm, entsprechend dem Elohistenbericht von Gen 2. Der Abschnitt endet mit der theologisch und anthropologisch relevanten Feststellung, dass die lebenserhaltende Seele, die der opifex ${ }^{43}$ Adam einhauchte, eine Neuschöpfung und keine Gottesspartikel war (Gen189-194 P.R.). Die dritte, gleichfalls auf dem Elohistenbericht (Gen 303-327 P.R.) beruhende Erzähleinheit behandelt die Erschaffung Evas aus der Rippe des schlafenden Adam. Dieser Schlaf Adams wird als Ekstase interpretiert, während derer er prophetische Visionen gehabt habe, die er später niederschrieb und veröffentlichte. Diese Legende ist ebenfalls der Historia scholastica zum Teil wörtlich, aber auf das Wesentliche gekürzt, entnommen (PL 198,1070 B). ${ }^{44}$ Abgeschlossen wird der Bericht über die Erschaffung Evas - entsprechend Isidors typologischer Erklärung in den Quaestiones in vetus testamentum (PL 83,217B) - mit einer Allegorie betreffend die Erschaffung der Kirche aus der Seitenwunde Christi bei seinem Tod am Kreuz, die ihm nach Jh 19.34 erst zugefügt wurde, als er bereits tot war - der Tod gilt ja bereits in der griechischen Mythologie als Bruder des Schlafes, d.h. als diesem wesensverwandt und daher mit ihm austauschbar. Dieser Umstand ist übrigens auch im ,Paradies' der Antike wirksam: Im Goldenen Zeitalter trat nach Hesiod, erg. 110-125, der tiefe Schlaf an die Stelle des Todes. Zurück zur Genesis: Der ,Neue (,letzte‘, ,endgültige‘) Adam‘, Christus (1 Kor 15.45), bringt durch seinen Tod die Kirche als ,neue (zweite, ,letzte‘, ,endgültige') Eva’ hervor, die den Sündenfall der ,ersten‘, während Adams Schlaf geformten, wieder rückgängig macht.

Als Abschluss einige Bemerkungen zu Darstellung der Jungfrau Maria in der Aurora. Es fällt auf, dass P.R. in keinem der drei Teile über die Erschaffung der Protoplasten näher auf deren Aussehen eingeht, obwohl es diesbezügliche Ansätze in der bibelepischen Tradition gab: Dracontius beschrieb die eben erschaffene Eva in ihrer nackten Schönheit und verglich sie sogar mit einer Meeresnymphe (laud. dei I.393-

43 Diese Übersetzung des „Handwerksgottes“, $\delta \eta \mu$ ıopyóc, aus Platons Timaios und „Ursprungs der besseren (d.h. aus dem Chaos heraus geordneten) Welt" erinnert an Ov. met. 1.79: ille opifex rerum, mundi melioris origo. Ovid verwendet das Wort wie P.R. auffälligerweise erst im Zusammenhang mit der Menschenschöpfung. Die im Haupttext erwähnte Definition der Menschenseele durch P. R. als einer Neuschöpfung ohne göttlichen Anteil dürfte als Verwahrung gegen die von Ovid an dem angeführten Abschnitt erwogene Möglichkeit der ,himmlisch-ätherischen` Herkunft des eben erschaffenen Menschen im Sinn der Stoiker (met. I.78: sive hunc [sc. hominem] divino semine [sc. deus] fecit ; I.80 - 81: sive recens tellus ... cognati retinebat semina caeli angebracht sein.

44 Gen 304 P.R.: non sompnus sed erat extasis ille sopor, vgl. hist. scholast.: immisit Deus soporem in Adam, non somnum, sed extasin; Gen 306 P.R.: et evigilans inde propheta fuit, vgl. hist. scholast.: et evigilans prophetavit; Gen 310 P.R.: inque suis libris hec reseravit, vgl. hist. scholast.: et libris suis postea indicavit. - Zur Tradition des Testaments Adams s. Knittel (2002); Adam gilt auch im Islam als der erste Prophet, s. https://de.wikipedia.org/wiki/Adam_im_Islam. 
397). ${ }^{45}$ P.R. dagegen erwähnt erst in der Einleitung zum Sündenfall kurz die schönen Leiber der Ureltern, ditabat singula membra decor (Gen 329 - 330 P.R.), ${ }^{46}$ aber eben die Schönheit der Körper, denen zwar noch jegliches sexuelle Verlangen fehlte, deren Liebreiz aber nach dem Genuss der verbotenen Frucht Ursache für Sexualität und deren negative Folgen für Leib und Seele war. Somit erscheint körperliche Schönheit von vornherein in negativem Licht. Dem von Eva verursachten Sündenfall setzt P.R. im Sinn des so genannten Protoevengliums, Gen 3.15, die verheißene Erlösung der Menschheit von ihrer Urschuld durch eine jungfräuliche Geburt entgegen - zur Hervorhebung dieses soteriologisch zentralen Satzes wendet er in beiden Versen das rhetorische Mittel der dreifachen Alliteration zweier Laute an: vipera vim perdit, sine vi pariente Puella / exclusit virus nescia Virgo viri (Gen. 361-362 P.R.), in Anlehnung an die auf Klangähnlichkeit von vipera und vis beziehungsweise von párere und dem Wortbestandteil -pera beruhende Etymologie des Wortes Viper, vipera, bei Isidor, orig. 12.4.10: vipera dicta quod vi pariat. ${ }^{47}$

Das in Gen 361 P.R. angeschnittene marianische Thema greift der Dichter erst einige Zeit später, in der zweiten Fassung der Aurora, mit einem vorbereitenden Abschnitt De pulcritudine Beate Virginis Marie auf (Ev 31-68), und zwar in Form einer Ekphrasis, die der Beschreibung der Schönheit des jugendlichen Absalom in Aurora, II Regum 41-72 nachgebildet ist, woraus sogar ganze Verse übernommen werden. ${ }^{48}$

45 Drac. laud. dei I.393-396: Constitit ante oculos nullo velamine tecta, / Corpore nuda simul niveo quasi nympha profundi. / Caesaries intonsa comis, gena pulchra rubore, / Omnia pulchra gerens, oculos, os, colla manusque. Dracontius evoziert die Ekphrasis der nackten Corinna nach Ov. am. I.5.17-22 durch das erste Hemistich von 1.393, wobei er Ovids Wortlaut: ut stetit ante oculos durch einen ähnlich lautenden, an drei anderen Stellen Ovids belegten (am. III.5,10, epist.15.162, 16.61: constitit ante oculos) ersetzte und den Vergleich mit der Meeresnymphe dem an zweiter Stelle angegebenen Ovidvers entnahm, worin eine Najade Subjekt ist. Durch die Bezugnahme auf Ovids Geliebte erhält Eva schon vor dem Sündenfall die Konnotation der erotischen Verführerin.

46 Den Ausdruck membra movere gebraucht mit Vorliebe Augustinus in seinen antipelagianischen Schriften als Euphemismus für sexuelle Betätigung (ausgehend von männlicher Aktivität), z. B. pecc. orig. (= grat. Christ. 2) 34.39; 36.41 (in Zusammenhang mit der Bedeckung der Genitalien nach dem Sündenfall); 35.40; nupt. et concup. I.24.27. Vgl. Anm. 41.

47 Den Hintergrund der Etymologie bietet ein erstmals bei Herodot, 3.109 berichtetes zoologisches Phänomen: Die Mutterschlange werde bei der Geburt ihrer Jungen von diesen ,gesprengt‘, die dadurch ihren Vater rächen, dem das weibliche Tier bei der oralen sexuellen Vereinigung den Kopf abgebissen habe. In der christlich-lateinischen patristischen Dichtung vgl. die indirekt Bezugnahme bei Marius Victor(ius). aleth. 1.505-507, dazu s. Smolak (1980) 181-188 mit weiteren Belegen aus der lateinischen patristischen Literatur.

48 Hier die Parallelstellen: zeigt Ähnlichkeit an, = Gleichheit: Ev $35 \sim$ II Regum 41 P.R.; 36 42; 38 43; 39 45; 40=46; 49= 51; 50=52; 51= 53; 52=54; 57=55; 58=56; 59=59; 60=60; 53=61; 54=62; 63=57; 64=58; 65=67; 66=68; 67=71; 68=72. - Einen Sonderfall stellen die Verse Ev und II Regum 66 P.R. dar (Beschreibung des natürlich schönen Gesichtes Marias): non hospes colit ( incolit) ora color, nec fuscus adulter (/ Inclita nature dona iuvare potest) (Absque pilis candor in colli colle diescit /) Nec niveum fuscat umbra pilosa decus (vom unbehaarten Nacken Absalos): Aus Gründen der Ausgewogenheit des Verses Ev 61 wäre die Rahmung zweier sinnverwandter Subjekt-Substantiva für Schminke durch je ein adjektivisch-attributiv gebrauchtes Substantiv (hospes, adulter: beide Begriffe bezeichnen in einer 
Diese Fortsetzung der im Genesisteil angelegten narrative Einheit beginnt schon im ersten Distichon von De pulcritudine mit Erwähnung der Aussendung Gabriels zu Maria (Ev 31-3249), wird mit Ev 33 durch die eigentliche Beschreibung und von Vers Ev 69 an durch eine bis Ev 134 reichende katalogartige Aufzählung der Verweise auf Maria in der gesamten Bibel, De commendatione Beate Marie Virginis in eloquiis scripturarum, unterbrochen und erst nach deren Ende mit der Einheit Ev 135-140 weitergeführt. Dass in Vers Ev 33 eine neue Einheit, eben die Ekphrasis, beginnt, markiert P.R. durch eine Apostrophierung seiner selbst: Hic, Petre, fige stilum; tam clare Virginis ortum, / virtutes, speciem carmine pinge novo (Ev 33-34): Er werde also unter Zuhilfenahme aller seiner sprachlichen Möglichkeiten hier ein „neues Lied“, carmine novo - in Anlehnung an den aus den Psalmen bekannten Terminus canticum novum, z.B. ps. 32.3 - vortragen, wobei sich die Neuheit auch auf den Umstand beziehen lässt, dass das Stück im Evangelienteil der Aurora erst in einem späteren Arbeitsgang dazugekommen ist. Die Aufforderung des Dichters an sich selbst betrifft ausschließlich die literarische Form, es gibt keine Allegoresen oder typologische Interpretationen in der Beschreibung der physischen Schönheit.

Die Ekphrasis folgt nach Angabe des Autors den entsprechenden Grundregeln: $\mathrm{Zu}$ behandeln seien ortus, virtutes, species (Ev 33-34). Als Schöpferin des materiellen Leibes Marias wird nicht Gott, sondern die im Mittelalter wie eine Göttin personifizierte Natura angegeben. ${ }^{50}$ Sie habe sich bei Maria so sehr verausgabt, dass es keine

Antiklimax ,Fremdheit') zu erwarten - der Gebrauch des Wortfeldes von adulter im Zusammenhang mit Damenkosmetik ist in der patristischen Literatur häufig belegt (z. B. Cypr. hab. virg. 14-15, laps. 6; Ambr. Cain et Ab. 1.4.14, 5.15; Aug. doctr. Christ. 4.21); als zweites Subjekt würde sich das metrisch taugliche fucus anbieten, an dessen Stelle unter dem Einfluss des Verbs fuscat aus der Ekphrasis Absaloms das Adjektiv fuscus getreten ist und adulter personifiziert erscheint: Der „dunkle Ehebrecher" muss sich auf die unter der Verwendung von Ruß hergestellte Lidschattencreme beziehen, die rötliche Gesichtsschminke verbirgt sich hinter dem unspezifischen color.

49 Ev 31-32: Post missus Gabriel in sexto mense (sc. nach der Empfängnis Johannes des Täufers), salutem / Affert angelicam, Virgo Maria, tibi. Durch das in der Dichtung häufig angewendete Stilmittel der namentlichen Apostrophierung einer Person oder Sache zwecks Fokussierung übernimmt P.R. gewissermaßen den Part des Verkündigungsengels: Das Distichon enthält eine Anspielung auf das Angelusgebet, die Selbstanrede in unmittelbarem Anschluss an die Apostrophierung Marias suggeriert dem Leser das Nahverhältnis eines Dialogs zwischen Dichter und seinem ,Gegenstand‘.

50 Die ,Göttin Natura‘ als Dienerin Gottes im Bereich der materiellen Weltschöpfung und -erhaltung wird seit Curtius (1948) 116-137 als handelnde Person im mittelalterlichen Weltbild, das letztlich auf hellenistischen Physis-Konzepten beruht, wahrgenommen. Dies wird besonders deutlich in der hochund spätmittelalterlichen Buchmalerei, welche die personifizierte Natura als kunstvolle Handwerkerin und Produzentin (vgl. Ev 133) noch nicht beseelter Kinderkörper mit Hammer, Amboss und gegebenenfalls anderen Schmiedewerkzeugen darstellt, s. Modersohn (2003) 86-92: Natura als Schöpferin und Künstlerin, dazu Abbildungen 2, 9-15. - In der Edition von Beichner (1965) ist natura in dem zu analysierenden Abschnitt allerdings durchwegs mit kleinem Anfangsbuchstaben geschrieben, das Wort sollte aber als Eigenname aufgefasst und daher mit großem Anfangsbuchstaben geschrieben werden: Denn an sämtlichen Stellen erscheint Natura aktiv beziehungsweise als Person: 35: Tanto compluit hanc (sc. Mariam) dives Natura decore (als Folge wundert sich Natura über ihre Verarmung); 39: s. Haupttext; 61: inclita Nature dona die von der Natur gegebene Schönheit könne kein ,Fremdling‘, 
zweite Frau dieser Art gab und sie selbst mittellos wurde. Diesen vom physischen Aspekt von Marias Einzigartigkeit ausgehenden Vorzug leitet P.R. in einen Lobpreis ihrer Tugend über, und zwar, wie zu erwarten, ihrer Jungfräulichkeit. Diesen Lobpreis handelt er durch einen Vergleich ihrer jungfräulichen Mutterschaft mit dem - im Mittelalter überwiegend als weiblich aufgefassten - Vogel Phönix ab, der ohne gleichen ist, wie sie, und sein Leben ebenfalls ohne Partner weitergibt. Die viel gerühmte, hier nicht ausgesprochene, aber selbstverständliche Schönheit des / der Phönix führt die narratio wieder zu der Fortsetzung der die leiblichen Vorzüge betreffenden Ekphrasis zurück, so dass das Lob der Partnerlosigkeit, für P.R. eine moralische Qualität, innerhalb dieser unorganisch wirkt. ${ }^{51}$ Denn die nun folgende Beschreibung ist gänzlich den Vorzügen von Marias äußerlicher Erscheinung gewidmet, so sehr, dass ihre ,Makellosigkeit‘ in Ev 39: unam nec maculam natura reliquit in ista, sich nicht auf die Jungfrau als immaculata in mariologischem Sinn bezieht, sondern auf ihren Leib: Die engste Parallele findet sich einmal mehr in Ovids Beschreibung der nackten Corinna, an deren Körper nicht der geringste Makel zu sehen war: in toto nusquam corpore menda fuit (am. I.5.18) - der Vers ist derselben erotische Elegie Ovids entnommen, die Dracontius für die Ekphrasis Evas herangezogen hatte. ${ }^{52}$ Was in der Aurora auf diese aus Ovid übernommene Feststellung folgt, ist eine kunstgemäße Beschreibung des weiblichen Körpers - im Fall der ,Neuen Eva' des bekleideten, selbstverständlich, was in gewissem Widerspruch zu der ovidischen Ankündigung des physischen Makellosigkeit steht. Die Ekphrasis Marias enthält typische Vergleiche, typische Details wie das harmonische Zusammenspiel von Rot und Weiß, die glatte Haut, das blonde Haar und die Überbietung indischen Elfenbeins durch Marias Zähne. Für modernes Empfinden befremdlich ist allein der Vergleich der Beine der ideal geformten Jungfrau mit Säulen in Ev 68: More columpnarum firmus uterque pedum. Der Vergleich ist wortwörtlich aus der Beschreibung Absalons übernommen, wo er die körperliche Tüchtigkeit des Jünglings charakterisiert, im Zusammenhang mit einer Frau muss er eher auf den geraden Wuchs und den aufrechten Gang abzielen.

Der bereits erwähnte, auf die Ekphrasis folgende Abschnitt über Marias Gegenwart in den Büchern der gesamten Bibel der Christen (Ev 69-134), beginnend mit der Arche Noah, wirkt zunächst wegen seines unerwarteten persönlichen Schlusses (Ev

61: hospes (color), und kein ,dunkler Ehebrecher', 61: (fuscus) adulter vermehren. Die Personifizierung der Kosmetik lässt Natura noch deutlicher als Person hervortreten.

51 Ev 35-38; der / die Phönix aufgrund seiner / ihrer sexuellen Indifferenz als marianisches Symbol findet sich z. B. bei Gauterus de Wymburnia (Winborne), hymnus Mariae, Str. 1.2-3: Que pudore meruisti / Dici fenix virginum. - Dass der Vogel in der Ekphrasis Marias bei P.R. in unvermutetem Kontext erscheint, erklärt sich aus der adaptierten Übertragung der Beschreibung der einzigartigen leiblichen Schönheit Absalos von II Regum 44 P.R.: (Pre cunctis pueris, quos ornat gratia forme) / Felix et phenix iste fit absque pare auf die moralische Schönheit Marias auf der Grundlage einer bereits vorhandenen Symbolik in Ev 38.

52 Es ist nicht anzunehmen, dass P.R. sich auf diesen Dichter aus dem Africa der Vandalenzeit bezog, dessen Werke er offensichtlich nicht kannte. Vielmehr zählte Ov. am. I.5 im Mittelalter zu den meistzitierten erotischen Texten und lag sogar in Sonderüberlieferung vor. 
129-134) nicht passend. In diesem drückt nämlich der Dichter seinen Wunsch aus, Marias Schönheit jetzt, nunc, zu schildern - obwohl er in Ev 33-68 bereits eine entsprechende Beschreibung geboten hatte, wenngleich erst in der überarbeiteten Version. Doch während er sich dort durch die Apostrophierung seiner selbst zu einem hohen Stil aufgefordert hatte (Ev 33-34), wünscht er an der späteren Stelle, dass die Jungfrau seinen unschönen Stil, deformem stilum (Ev 130) - die Worte stehen in deutlichem Gegensatz zu fige stilum in Ev 33 - kraft ihrer Schönheit ziere, wie er in einem ein wenig krampfhaft wirkenden Spiel mit der Wortfeld forma erbittet. Da die strahlende Schönheit von Marias Antlitz, die mit der Morgenröte in Wettstreit stehe (Ev 134), menschliches Maß überschreite und „gottgleich“, numinis instar ${ }^{53}$, sei (Ev 132), lasse sie die Gaben der ,Künstlerin Natura‘, artificis Nature (Ev 133), das bedeutet: Jene, die $\mathrm{zu}$ beschreiben er in der vorangehenden Ekphrasis (Ev 33-68) noch mit ,menschlicher Sprache fähig gewesen war, armselig erscheinen (Ev 129-134). ${ }^{54}$ Nunc in Ev 129 bezeichnet also die nunmehr gegenüber der vorangehenden Ekphrasis grundsätzlich veränderte Voraussetzung einer Beschreibung. Mit der Metapher der Morgenröte für Marias Antlitz in Ev 134 spielt P.R. offenkundig auf den Titel seines Werkes, Aurora, unter Anwendung des Endreimeffekts von (certans) aurore und or-is (hon-or) an - eine raffinierte, schon in dem eben zitierten Vers Ev 130 vorbereitete Variante der Topoi der Bescheidenheit beziehungsweise des helfenden Eingreifens eines ,übermenschlichen' Wesens in den formal-ästhetischen Akt dichterischen Schaffens. ${ }^{55}$ Vor allem aber rechtfertigt die Aussage der Verse Ev 129-134 das Fehlen einer detaillierten Beschreibung Marias an dieser Stelle. Betrachtet man den Abschnitt über Marias Präsenz in der Bibel (Ev 69-128) im Zusammenhang mit der ausführlichen Verkündigungsszene in Ev 135-140, Missus ad hanc Gabriel etc., einer Doublette der knappen von Ev 31-32, so zeigt sich, dass in Ev 135 ein Anschluss an diese glatt verliefe. Denn bereits Ev 128 lautet: Cuius (sc. Virginis) in exortu lux fuit orta reis, „(die Jungfrau), bei deren Aufgang - man beachte die astronomische Metapher des Aufgangs für Maria, die stella maris beziehungsweise den Morgenstern - das ,Licht“ (nämlich Christus als Sol iustitiae beziehungsweise als „Licht der Welt“, vgl. Jh 8.12, oder „Sonne der Gerechtigkeit“, Mal 3.20 [= 4.23]) - für die Sünder aufgegangen ist.“ Die Verkündigungsszene von 135 würde also sprachlich und inhaltlich bruchlos anschließen. Wenn der Dichter aber die Verse in der zweiten Fassung, in die er eine

$53 \mathrm{Zu}$ der Wortverbindung numinis instar vgl. Ov. am. III.11.47: Der Kontext ist ebenfalls Frauenschönheit: perque tuam faciem (vgl. oris bei P.R.), magni mihi numinis instar (dieser Halbvers wird in der nachklassischen Dichtung mehrmals zitiert), met. XIV.124: numinis instar eris semper mihi (in ähnlichem Kontext).

54 Ev 129-134: Nunc metra formare de forma Virginis opto; / Ornet deformem Virgo decora stilum. / Humanam speciem transcendit forma puelle; / Excedens hominem, numinis instar habet. / Pauperat artificis nature (Nature Smolak) dona, decori / Certans aurore, splendidus oris honor. Zum Konzept der sich bei der Schöpfung eines besonderen menschlichen Individuums verausgabenden Natura vgl. Alan., Anticlaud. 7.12 (den Hinweis danke ich Christine Ratkowitsch, Wien).

55 Vgl. in der christlichen Dichtung der Spätantike z. B. Paul. Nol. carm. 23.27-36. 
umfangreiche Ekphrasis der leiblichen Schönheit einbaute, trotzdem nicht tilgte, so konnte er seine Zurückweisung einer solchen an der späteren Stelle (Ev 129-134) immerhin noch zusätzlich damit rechtfertigen, dass er in den unmittelbar vorangehenden Versen Maria als die von der Sonne umhüllte und von zwölf Sternen bekränzte himmlische Frau der Apokalypse nach Off 12, präsentiert hatte (Ev 125-128), die sich infolge ihre übernatürlichen Wesens, wie bereits erwähnt, jeder natürlichen Beschreibung entziehe.

Die ergänzte Ekphrasis der physischen Schönheit in Ev 31-68 scheint dagegen dreifach motiviert zu sein. Erstens: P.R. konnte Maria als die Neue Eva präsentieren, in ihrer vollen natürlichen, für alle Zeiten unverdorbenen und nie korrumpierbaren Schönheit - die Schönheit der 'Mutter des Lebens' schien in der Exegese außer Frage zu stehen. Das Konzept einer ,umgedrehten Eva' in Analogie zu Christus als dem Neuen (zweiten) Adam (1 Kor 15.45) war für die lateinische Welt seit dem frühen Mittelalter allein in dem Gruß des Engels ausgesprochen: ave ist ja, anagraphisch gelesen, die Umkehrung von Eva, worauf P.R. schon Gen 323-324 in der Darstellung des Sündenfalls Bezug genommen hat. ${ }^{56}$ Der zweite Grund für die Einfügung der ersten Ekphrasis dürfte darin liegen, dass jede Beschreibung die Gelegenheit bietet, ein typisches Produkt rhetorischer Schulbildung mustergültig vorzuführen, hier technisch besonders reizvoll, da es galt, die Beschreibung einer männlichen Person auf eine weibliche umzulegen. Drittens: schließlich entsprach es vermutlich dem Wunsch des Dichters, in sein Werk ein zeitgenössisches poetisches Sujet einzuschließen, die lyrische Marienminne, die spiritualisierte Form der höfischen Minnedichtung. ${ }^{57}$ Eine mögliche Parallele zu diesem Streben von P.R. nach Selbstdarstellung als Künstler kann darin gesehen werden, dass er in die außerbiblische Geschichte des Schicksals des siechen Herodes nach dem Bethlehemitischen Kindermord und der Hinrichtung des Antipater in Ausarbeitung einer vergleichsweise knappen Angabe in der Historia Scholastica (PL 198,1546C-1548 A) ein kunstvolles Paar langer juristischer Reden und Gegenreden vor Kaiser Tiberius in Rom einbaute (Ev 479-778), ebenfalls Musterstücke der Schulrhetorik und vergleichbar mit den Reden von Ajas und Odysseus im Streit um die Waffen Achills, die auf der Grundlage von Ovid, met. XII.620XIII.382, ein mittelalterlicher Anonymus verfasste. ${ }^{58}$

Hier wäre die Stelle, einen Blick auf die gelehrten, mitunter gewagten und aus heutiger Sicht nicht immer sachlich zutreffenden Erweiterungen des Textes der Aurora durch Aegidius zu werfen, dessen Tätigkeit mehrfach bereits kurz erwähnt wurde. Dies würde aber den Rahmen vorliegender Untersuchung sprengen, deren Ziel es war, anhand der Analyse einiger weniger Abschnitte aus dem komplexen Werk der Aurora deutlich zu machen, welch umfangreiche philologisch-exegetische Arbeit an jenem

56 Gen 323-324 P.R.: Eva necem mundo dedit; hoc nomen retroverte, / Fiet ave, per quod fulsit in orbe salus.

57 Zu Marienminne vgl. Nemes (o.J.); Stanovská (2003) 117-130; Frings (1960) 20.

58 Herausgegeben von Schmidt (1964) 100 -132; zur stilkritischen Interpretation s. Smolak (2004b) 511- 524 . 
im Spätmittelalter so hoch geschätzten Verskommentar zu großen Teilen der Bibel zu leisten wäre. Das gilt auch und vielleicht noch mehr für die Rezeptionstexte, und zwar nicht bloß für die zweistufige Erweiterung durch Aegidius, sondern auch für die zahlreichen, in weit gestreuter Überlieferung erhaltenen kleineren Stücke. Mit der Erweiterung von deren Bestand ist zu rechnen, wie die diversen Funde durch die verdienstvolle, unermüdliche Forschungstätigkeit von Greti Dinkova-Bruun vermuten lassen. ${ }^{59}$ Die Aurora ist somit mutatis mutandis bis heute ein opus operantium, ein work-in-progress.

\section{Literaturverzeichnis}

\section{Edition}

Beichner (1965): Aurora. Petri Rigae Biblia Versificata. A Verse Commentary on the Bible, Part I, Part II, ed. Paul E. Beichner, C.S.C., Notre Dame (Indiana) 1965.

\section{Sekundärliteratur}

Arweiler (1988): Alexander Arweiler, Die Imitation antiker und spätantiker Literatur in der Dichtung ,De spiritalis historiae gestis' des Alcimus Avitus. Mit einem Kommentar zu Avit. carm. 4, 429-540 und 5, 526-703, Berlin.

Buschhausen (1987): Helmut Buschhausen, „Die Geschichte der Inschriften auf dem Verduner Altar des Nikolaus. Überlegungen zu einer Neudatierung“, in: Wiener Studien 100, 265-309.

Buschhausen (1980): Helmut Buschhausen, Der Verduner Altar, Wien.

Curtius (1948): Ernst Robert Curtius, Europäische Literatur und lateinisches Mittelalter, Bern.

Dinkova-Bruun (2016): Greti Dinkova-Bruun, „Verse Epigrams Added to Peter Riga's Aurora from Olomouc“, in: Sacris Erudiri 55, 391-418.

Dinkova-Bruun (2008a): Greti Dinkova-Bruun, „Rewriting Scripture: Latin Biblical Versification in the Later Middle Ages“, in: Viator 39,1, 263-284.

Dinkova-Bruun (2008b): Greti Dinkova-Bruun, „“Proverbia Salomonis“. An Anoymous Accretion to Peter Riga's „Aurora““”, in: Frank T. Coulson, Anna A. Grotaus (edd.), Classica et Benevantana (Studia in onore di Virginia Brown), Turnhout, 9-44.

Dinkova-Bruun (2007): Greti Dinkova-Bruun, „Additions to Peter Riga’s „Aurora“ in Paris. Bibliothèque Nationale de France, lat. 13050“, in: Medieval Studies 69, 1-57.

Dinkova-Bruun (2006): Greti Dinkova-Bruun, „Peter Rigả s Aurora and its Gloss from Salzburg, Stiftsbibliothek St. Peter, MS a VII.6“, in: G. B. Wieland, C. Ruff, R .G. Arthur (edd.), Insignis Sophiae Arcator. Medieval Latin Studies in Honour of Michael Herren on his $65^{\text {th }}$ Birthday, Turnhout, 237-260 (Publications of the Journal of Medieval Latin 6).

Dölger (1936): Franz Dölger, „Lumen Christi“, in: Antike und Christentum 5,1,1-43.

59 Dinkova-Bruun (2016), 391-418; Dinkova-Bruun (2008b) 9-44; Dinkova-Bruun (2007) 1-57; vgl. auch Anm. 10. 
Donnini (1995): Marco Donnini, „Versificazione: I testi“, in: Guglielmo Cavallo, Claudio Leonardi, Enrico Menestò (edd.), Lo spazio letterario del medioevo III: La ricezione del testo,1. Il Medioevo Latino, Rom.

Fillitz, Pippal (1987): Hermann Fillitz, Martina Pippal, Schatzkunst, Salzburg - Wien 1987.

Frings (1960): Theodor Frings, „Die Anfänge der europäischen Liebesdichtung im 11. und

12. Jahrhundert", in: Sitzungsberichte der Bayerischen Akademie der Wissenschaften 1960, Bd. 2, 1-23.

Green (2006): Roger P. H. Green, Latin Epics of the New Testament. Iuvencus, Sedulius, Arator, Oxford.

Heikel (1902): Ivar v. Heikel, Eusebius, Erster Band, Leipzig (Die griechischen christlichen Schriftsteller der ersten drei Jahrhunderte).

Herren (2007): Michael Herren, „Reflections on the Meaning of the Ecloga Theoduli: Where is the Authorial Voice?“, in: Vigiliae Christianae, Suppl. 87, 190-230.

Herzog (1975): Reinhart Herzog, Die Bibelepik der lateinischen Spätantike. Formgeschichte einer erbaulichen Gattung, Band 1, München.

Hofmann, Szantyr (1965): Johann Baptist Hofmann, Anton Szantyr, Lateinische Grammatik, zweiter Band, München (Handbuch der Altertumswissenschaft, 2. Abteilung, 2. Teil, 2. Band).

Kartschoke (1975): Dieter Kartschoke, Bibeldichtung. Zur Geschichte der epischen Bibelparaphrase von Juvencus bis Ottfried von Weißenburg, München.

Knittel (2002): Thomas Knittel, Das griechische 'Leben Adams und Evas', Tübingen.

Leithe-Jasper (2015): Helena Leithe-Jasper, „Versus ad domus domini Mogontine. Mehr als nur eine tituli-Sammlung?“, in: Nobert Kössinger, Elke Krotz, Stephan Müller (Hrsg.), Ekkehart IV von St. Gallen, Berlin - Boston, 373-391 (Lingua Historica Germanica 8).

Loerke (2003): Marc-Oliver Loerke, Höllenfahrt Christi und Anastasis. Ein Bildmotiv im Abendland und im christlichen Osten, Regensburg (Dissertation).

Modersohn (2003): Mechthild Modersohn, „Natura als Göttin - eine Personifikation zwischen Mythos und Aufklärung“, in: Peter Dilg (ed.), Natur im Mittelalter. Konzeptionen - Erfahrungen - Wirkungen. Akten des 9. Symposiums des Mediävistenverbandes, Marburg, 14.-17. März 2001, Berlin, 84-110.

Nemes (o.J.): Balázs J. Nemes, „minne - vrouwe - Maria. Interferenzen zwischen Marienlyrik und Liebeslyrik im Hoch- und Spätmittelalter“: https://www.academia.edu/19112799/minne_ vrouwe_Maria_Interferenzen-zwischen-Marienlyrik_und_Liebeslyrik (abgerufen 29.3.2019)

Oppermann (1926): Hans Oppermann, „Petrus Riga und Petrus Comestor“, in: Zeitschrift für Romanische Philologie 4,1, 55-73.

Petringa (2016): Maria Rosaria Petringa, Il poema dell'Heptateuchos, Catania.

Roberts (1985): Michael Roberts, Biblical Epic and Rhetorical Paraphrase in Late Antiquity, Liverpool.

Ruf (2011): Martin G. Ruf, Die heiligen Propheten, eure Apostel und ich. Metatextuelle Untersuchungen zum zweiten Petrusbrief, Tübingen 2011.

Schmidt (2001): Paul Gerhard Schmidt, „La Bibbia versificata: I testi mediolatini“, in: Francesco Stella (ed.), La scrittura infinita. Bibbia e poesia in età medievale e umanistica, Firenze, $449-457$.

Schmidt (1964): Paul Gerhard Schmidt, „'Causa Aiacis et Ulixis I-Il'. Zwei ovidianische Streitgedichte des Mittelalters", in: Mittellateinisches Jahrbuch 1, 100-132.

Schwind (1990): Johannes Schwind, Arator-Studien, Göttingen 1990.

Smolak (2015): Kurt Smolak, „The 'epigrammatic' Epic. Ekkehart's Versus ad picturas and the Poetic Tradition“, in: Symbolae Philologorum Posnaniensium XXV/2, 73-95.

Smolak (2004a): Kurt Smolak, „Maro mutatus in melius? Zum Phänomen des literarischen Zitierens in der christlich-lateinischen Spätantike“, in: Oswald Panagl, Ruth Wodak (Hrsg.), Text und 
Kontext. Theoriemodelle und methodische Verfahren im transdisziplinären Vergleich, Würzburg, 51-66.

Smolak (2004b): Kurt Smolak, „Semivir arma tulit. Zu den mittellateinischen 'Causae Aiacis et Ulixis'“, in: Paul Gerhard Schmidt, Andreas Bihrer, Elisabeth Stein (Hrsg.), Nova de veteribus. Mittel- und neulateinische Studien für P. G. Schmidt, München, Leipzig, 511-524.

Smolak (2001, 1999): Kurt Smolak, „Die Bibeldichtung als „Verfehlte Gattung“““, in: Francesco Stella (ed.), La scrittura infinita. Bibbia e poesia in età medievale e umanistica, Firenze 2001, 15-29 (mit geringfügigen Abweichungen bereits gedruckt in: Wiener Humanistische Blätter 41 [1999], 7-24).

Smolak (1994): Kurt Smolak, „Horazische Lyrik zwischen Ablehnung, Anverwandlung und Travestie. Ein Rezeptionsparadigma“, in: Janus 15, 26-38.

Smolak (1980): Kurt Smolak, „Die Geburtsschmerzen Evas bei Claudius Marius Victorius“, in: Grazer Beiträge 9, 181-188.

Stanovská (2003): Sylvie Stanovská, „“Es strahlt ein Licht aus der Wolke“. Zur Figur der Frau in der mittelalterlichen Literatur als Anlass von Begriffs-, Motiv- und Gattungsmischungen“, in: Sborník Prací Filosofické Fakulty Brněské Univerzity 8, 113-130.

Stella (2001): Francesco Stella (ed.), La scrittura infinita. Bibbia e poesia in età medievale e umanistica, Firenze.

Thraede (1962): Klaus Thraede, „Epos“, in: Reallexikon für Antike und Christentum 5, 983-1042. 